\section{POBREZA, DESIGUALDAD SOCIAL Y VULNERABILIDAD A LOS DESASTRES EN VENEZUELA}

Bettys Farias $^{1 *}$, Adriana Marquez ${ }^{1}$, Edilberto Guevara ${ }^{1}$

\section{RESUMEN}

El objetivo de esta investigación es proponer la noción de la pobreza, la desigualdad social y la privación, como elementos que causan vulnerabilidad a los desastres, demostrando la forma en la que la construcción social del riesgo se relaciona directamente con las políticas públicas nacionales de acceso a viviendas, empleos, y ordenamiento territorial. Esta investigación es de tipo descriptiva, el diseño es un trabajo de campo donde la información se obtiene mediante la aplicación de entrevistas, encuestas y observación directa, con actividades orientadas a la identificación de escenarios de riesgo como indicadores del nivel de pobreza de los sectores más desasistidos del país. Aporta reflexiones sobre las acciones que permitan contar con una población convenientemente informada, con recursos para una vida digna, con instituciones responsables y eficientes, propósitos que deben ser el direccionamiento de cualquier comunidad. Desde esta experiencia se concluye que la idea general sobre la vulnerabilidad a los desastres está estrechamente ligada a la pobreza extrema y la significativa desigualdad económica y social que existe en Venezuela.

\section{PALABRAS CLAVES}

Pobreza, Riesgo, Vulnerabilidad, Venezuela

\section{POVERTY, SOCIAL INEQUALITY AND VULNERABILITY TO DISASTERS IN VENEZUELA}

\section{ABSTRACT}

The objective of this research is to propose the notion of poverty, social inequality and deprivation, as elements that cause vulnerability to disasters, demonstrating how the social construction of risk is directly related to national public policies of access to housing, jobs, and territorial ordering. This research based on a fieldwork data where information was obtained through the application of interviews, surveys and direct observation. These activities aimed at identifying risk scenarios as indicators of the poverty levels of the most unassisted sectors of the country. Providing reflections on the actions that enabled to have a suitably informed population, with resources for a dignified life, with responsible and efficient institutions, the study highlights these actions as the direction of any community. From this experience, it is concluded that the general idea of vulnerability to disasters is closely linked to the extreme poverty and significant economic and social inequality that exists in Venezuela.

\section{KEYWORDS}

Poverty, Risk, Vulnerability, Venezuela
1. Centro de Investigaciones Hidrológicas y Ambientales, Universidad de Carabobo, Carabobo, Venezuela.

*Autor de correspondencia: bettysfarias@gmail.com

DOI:

https://doi.org/10.55467/ reder.v6i1.84

\section{RECIBIDO}

1 de febrero de 2021

\section{ACEPTADO}

4 de noviembre de 2021

\section{PUBLICADO}

1 de enero de 2022

\section{Formato cita}

Recomendada (APA): Farias, B., Marquez, A. \& Guevara, E. (2022). Pobreza, Desigualdad Social y Vulnerabilidad a los Desastres en Venezuela. Revista de Estudios

Latinoamericanos sobre Reducción del Riesgo de Desastres REDER, 6(1), 5165. https://doi.org/10.55467/ reder.v6i1.84

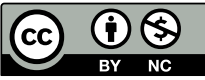

Todos los artículos publicados en REDER siguen una política de Acceso Abierto y se respaldan en una Licencia CreativeCommons Atribución-NoComercial 4.0 Internacional.

Revista de Estudios

Latinoamericanos sobre Reducción del Riesgo de Desastres (REDER)

Diseño: Lupe Bezzina 


\section{INTRODUCCIÓN}

La pobreza es un fenómeno muy complejo, heterogéneo y multidimensional, con muchas definiciones y basada en las diferentes teorías de las ciencias sociales (Rincón, 2014). La pobreza describe la situación de aquellos hogares que no logran reunir, en forma relativamente estable, los recursos necesarios para satisfacer las necesidades básicas de sus miembros (Feres y Mancero, 2001). Desde la perspectiva del desarrollo humano, la pobreza significa que se priven las oportunidades y las opciones más fundamentales del desarrollo humano: vivir una larga vida, sana y creativa, disfrutar de un nivel de vida decente, libertad, respeto por sí mismo, entre otros (PNUD, 2010).

La pobreza es un complejo de ataques a la dignidad humana. La devaluación del pobre como persona prepara el terreno para su discriminación, y, en última instancia, para su demonización (Klisberg et al., 2009), agrupando un conjunto de situaciones que están vinculadas con limitaciones para tener acceso o carecer de recursos para poder satisfacer las necesidades básicas (alimentación, educación, salud, vivienda, agua potable) que tienen incidencia sobre las personas (Phelean, 2006), ocasionando las desigualdades del desarrollo humano que tanto dañan las sociedades y debilitan la cohesión social y la confianza de la población en los gobiernos, las instituciones y sus congéneres (PNUD, 2019). Las desigualdades constituyen un obstáculo crucial para hacer realidad la Agenda 2030 para el Desarrollo Sostenible (NU, 2019; Lusseay y Mancini, 2019), estas desigualdades están reflejadas también en los daños ocasionados por los desastres, los cuales serán directamente proporcionales al nivel de desarrollo de la comunidad afectada (Maskrey, 1993; Lavell, 2004; Blaikie et al., 1996; Arrillaga et al., 2009) y además las diferencias entre grupos traban el desarrollo y son una barrera a la erradicación de la pobreza, la ampliación de la ciudadanía, el ejercicio de los derechos y la gobernabilidad democrática (CEPAL, 2016; CEPAL, 2018)

Son muchos los conflictos violentos, amenazas políticas, tensiones comunitarias, falta de atención a la salud pública, delincuencia, corrupción, discriminación y falta de capacidad de respuesta de las instituciones del estado que convierte a los más pobres en personas vulnerables (EIRD, 2009). Todo esto sin mencionar aun el aspecto a desarrollar en esta investigación que es su vulnerabilidad a las amenazas ambientales y a sus consecuencias.

En los estudios realizados por Blaikie y otros (1996), Cutter y otros (2000; 2003; 2009) y García (1993) se observa una coincidencia respecto de los factores de vulnerabilidad que recurrentemente contribuyen a la ocurrencia de los desastres: la pobreza, estrechamente relacionada con el nivel de desarrollo económico de los países; la expansión urbana hacia áreas con alto riesgo de inundaciones y deslizamientos de tierra; la creciente degradación del medio ambiente, tanto a escala local como global, hasta el cambio climático, responsable del aumento del nivel de mar y de importantes cambios en los regímenes de lluvias en todo el mundo; la crisis climática muestra que el precio de la inacción aumenta con el tiempo, ya que fomenta una mayor desigualdad (PNUD, 2019), que se ve reflejada, además, en la forma como la crisis climática está afectando ya de manera más acu $\urcorner$ sada a los más pobres, creando el espectro de un futuro incierto como consecuencia de dichos cambios (Saad, 2019). Los que poseen mayor vulnerabilidad social, es decir, que son incapaces de absorber los efectos de un determinado cambio en su medio ambiente (Wilches-Chaux, 1993), a su vez se enfrentan a mayores amenazas ecológicas como el estrés hídrico, sequías, inundaciones, ciclones, aumento de la temperatura y aumento del nivel de los mares (PNUD, 2020a). El hecho de vivir en la pobreza y con privaciones los mantiene atrapados en ciclos de baja productividad, desempleo estacional y bajos salarios, acentuando su vulnerabilidad a cambios en los patrones climáticos extremos (PNUD, 2011; PNUD, 2014), sobre todo a las inundaciones y sequías (CRED, 2013).

El Banco Mundial en su informe "La pobreza y la prosperidad compartida 2020", estima que a nivel mundial unos 1470 millones de personas viven en zonas con alto riesgo de inundaciones, entre ellas, alrededor de 132 millones de pobres, definidos según la línea internacional de la pobreza de USD 1,90 al día. Hallegatte y otros (2015) en la segunda de sus tres notas políticas extraídas de la obra Ondas de Choque: Contener los impactos del cambio climático en la pobreza, plantean que para 2030, el cambio climático si no se aborda podría empujar otros 100 millones de personas a la pobreza. 
Mientras que para el Banco Interamericano de Desarrollo (BID, 2020), las consecuencias del cambio climático y los eventos naturales exaltan la desigualdad social: i) porque en los países más pobres a menudo presentan más exposición a estos efectos climáticos; ii) los pobres sufren mayores pérdidas en proporción a su riqueza; y, iii) tienen escasos recursos para recuperarse y reciben menos apoyo de familiares, sistemas financieros y gobiernos (Hallegatte et al., 2017). Siendo tan desproporcionada la devastación que sufren los más pobres queda de manifiesto la dirección de causalidad: porque se es pobre, se es vulnerable (CEPAL, 2014; PNUD, 2014) y eso significa estar a menudo, más expuestos a los peligros naturales y al cambio climático (BID, 2020); están al menos dos veces más expuestos que los no pobres (Kim, 2012).

El ingreso y la riqueza están relacionados con la exposición a los eventos naturales y a los efectos del cambio climático de un país (BID, 2020). Los países más pobres han invertido considerablemente menos en protección contra amenazas naturales (Scussolini et al., 2016). La desigualdad en ingresos es una de las razones que ha provocado la situación venezolana que presenta un escenario turbulento en lo económico, político, social y cultural, atentando seriamente con la calidad de vida de los que viven el territorio, especialmente la clase media y baja que es muy representativa en la población total (Rincón, 2014), escenario que lo convierte en un país con altos índices de "pobreza extrema", siendo actualmente un desafío plantearse conceptos como desarrollo, calidad de vida, gestión de riesgos, sostenibilidad.

Según PNUD (2018) "Venezuela cae diez y seis puestos en el Indice de Desarrollo Humano", es el indicador que refleja los logros nacionales en materia de salud, educación y nivel de ingresos. El país ha sido calificado como más pobre de América Latina, incluso por encima de Haití (49,9\%); en el mundo solo Nigeria, con $77,6 \%$, supera a Venezuela en pobreza (Sinergia, 2020). De acuerdo a la Encuesta Sobre Condiciones de Vida en Venezuela de 2019-2020 (Encovi, 2020) se revela que la caída de $70 \%$ del Producto Interno Bruto (PIB), acumulada en los últimos siete años, deja a su paso $96,2 \%$ de la población en pobreza por ingresos, y a $79,3 \%$ en pobreza extrema y que no puede cubrir la cesta básica, con una inflación acumulada entre marzo de 2019 y marzo de 2020 que se ubica en $3365 \%$.

Aunado a todo lo expuesto anteriormente, la población venezolana al igual que la población de prácticamente todos los países y de todos los niveles de ingresos, se ve afectada por las consecuencias sanitarias y económicas de la pandemia de COVID-19. Sin embargo, los datos más recientes demuestran que la crisis golpea con especial intensidad a las personas que ya son pobres o vulnerables (PNUD, 2020a, BID, 2020). Esta pandemia puede haber sumido a unos 100 millones de personas en la pobreza extrema, el mayor retroceso registrado en una generación (PNUD, 2020b; PNUD y OPHI, 2020). En Venezuela, según encuestas de Encovi (2020), la crisis sanitaria por el COVID-19 ha generado que hasta $43 \%$ de los hogares reporten imposibilidad de trabajar o pérdida de ingresos. Los hogares que dijeron que trabajaron más horas pasaron de $41 \%$ a $49 \%$, quienes pidieron dinero a sus familiares pasaron de $30 \%$ a $35 \%$. De acuerdo a Sinergia (2020), Venezuela no está preparada para atender desastres ocasionados por el clima. Tanto aquellos que puedan relacionarse fácilmente, como sequías o inundaciones por temporadas de lluvias, como enfermedades o fallas de servicios públicos por consecuencia indirecta del clima.

Estas circunstancias son las que han motivado a la Universidad de Carabobo (UC) y del Centro de Investigaciones Hidrológicas y Ambientales (CIHAM-UC) a llevar a cabo esta investigación en seis comunidades del municipio Naguanagua, estado Carabobo, para abordar el tema de la pobreza, las privaciones y la desigualdad social como parámetros causantes de la vulnerabilidad a los desastres en estas comunidades. El tema de esta investigación reviste su importancia a nivel mundial en las metas del Objetivo 1 de Desarrollo Sostenible (ODS) de las Naciones Unidas, que propone erradicar la pobreza extrema para todas las personas y en todo el mundo a 2030; reducir al menos a la mitad la proporción de personas que viven en la pobreza en todas sus dimensiones; fomentar la resiliencia de los pobres y las personas que se encuentran en situaciones de vulnerabilidad y reducir su exposición y vulnerabilidad a los fenómenos extremos relacionados con el clima y otras perturbaciones y desastres económicos, sociales y ambientales (Naciones Unidas, 2018). Pero, además, el Marco de Sendai para la Reducción del Riesgo de Desastres 2015-2030, tiene entre sus objetivos: reducir de forma sustancial la mortalidad causada por desastres a nivel mundial y lograr ciudades y asentamientos humanos inclusivos, seguros, resilientes y sostenibles. 


\section{METODOLOGIA}

\section{Área de estudio}

Esta investigación se desarrolló en el estado Carabobo, ubicado en la región centro-norte de la República Bolivariana de Venezuela, integrado por 14 municipios, de los cuales se seleccionó el municipio Naguanagua, ubicado en la zona centro-norte del estado. Posee una superficie de 188 $\mathrm{km}^{2}$, representando un 4,3\% de la superficie de todo el estado. Según la proyección no publicada de la Alcadia del Municipio Naguanagua la población para el año 2019 era de 191.093 habitantes.

\section{Materiales y métodos}

La investigación se dividió en dos etapas: una correspondiente a la estimación del nivel de pobreza y otra a la identificación del escenario de riesgo de las comunidades.

\section{Estimación del nivel de pobreza}

La pobreza constituye un problema multifactorial, difícil de comprender globalmente. Considerando las limitaciones al modelarlo, esta investigación busca identificar algunos parámetros que permitan acercarse a su estimación y su relación con la vulnerabilidad a los desastres asociados a eventos naturales.

Dada la dificultad de medir algunos parámetros que forman parte de la calidad de vida, el estudio de la pobreza se ha restringido a aspectos cuantificables y obviamente de tipo material asociados al concepto de nivel de vida (Feres y Mancero, 2001).

Existen muchos métodos para identificar los hogares pobres, en este caso se aplicó el denominado método directo que evalúa directamente si los miembros de los hogares han logrado satisfacer sus necesidades básicas, consultándoles sobre los bienes y servicios de que disponen (Aponte et al., 2015), relacionando el bienestar con el consumo efectivamente realizado. Este método es conocido como Necesidades Básicas Insatisfechas (NBI), implementado por la Comisión Económica para América Latina y el Caribe (CEPAL) con la finalidad de analizar la información adquirida en censos demográficos y de vivienda (Feres y Mancero, 2001; Calvo y Giraldez, 2000; Battinson et al., 2007); esta medición de las NBI puede considerarse como una aproximación parcial al fenómeno de la pobreza (Fernández, 2012).

Para analizar la pobreza primero es necesario definirla, estableciendo los aspectos que abarca. Su medición requiere de indicadores cuantificables, que guarden relación con la definición seleccionada. La situación propia de un país es la que determina qué tan apropiado es un indicador. Existen ciertas carencias que se han constituido en el común denominador de las aplicaciones de este método: a) hacinamiento; b) vivienda inadecuada; c) abastecimiento inadecuado de agua; d) carencia o inconveniencia de servicios sanitarios para el desecho de excretas; e) inasistencia a escuelas primarias de los menores en edad escolar; y, f) un indicador indirecto de capacidad económica (CEPAL, 2001). Una vez elegidos los indicadores de necesidades básicas, es necesario establecer los umbrales de privación que definen la situación de carencias críticas: el umbral elegido debe corresponder a la mínima satisfacción posible de necesidades que sea compatible con una participación adecuada en la sociedad.

Generalmente, cuando un hogar presenta carencia en alguna de las dimensiones, éste se considera con NBI, por lo tanto, en estricto rigor este método permite medir el número de hogares que no ha satisfecho alguna necesidad básica, pero no necesariamente mide la pobreza. Esto se debe, entre otros factores, a que no existe una forma única y establecida de relacionar el número de NBI con la condición de pobreza, lo que implica que la clasificación final en pobres y no pobres es arbitraria y queda entregada enteramente al criterio del investigador.

En esta investigación se utilizó el criterio del Instituto Nacional de Estadística (INE) que plantea: una carencia en el hogar representa una necesidad básica insatisfecha, definiendo así los hogares pobres como aquellos que reportan al menos una de las necesidades básica insatisfecha, y como pobres extremos los que poseen dos o más necesidades básicas insatisfechas.

\section{Selección de la muestra}

Para la selección del número de entrevistas a aplicar en cada comunidad se empleó la ecuación que permite calcular el tamaño de la muestra (n), conociendo el tamaño de la población (N). 


$$
\begin{gathered}
\text { Ecuación } 1 \\
\mathrm{n}=\frac{\mathrm{N} \times \mathrm{Zc}^{2} \times \mathrm{p} \times \mathrm{q}}{(\mathrm{N}-1) \times \mathrm{e}^{2}+\mathrm{Zc}^{2} \times \mathrm{p} \times \mathrm{q}}
\end{gathered}
$$

Como un ejemplo de la aplicación de esta ecuación en la selección de una muestra representativa de la comunidad Colinas de Girardot I, con 229 viviendas, se utilizaron los siguientes valores para los parámetros de la Ecuación 1 (Arias, 2012).

Zc= nivel de confianza, para un 95\% el valor de Z es 1.96 (Suárez, 2012)

$p=$ Proporción de elementos que presentan una determinada característica a ser investigada, es decir probabilidad a favor. Para esta investigación esta variable define al porcentaje de la población cuyo representante de familia es de sexo masculino, partiendo del supuesto que los representes están distribuidos en porcentajes iguales, es decir $p=50 / 100=0.5$

$q=$ Proporción de elementos que no presentan la característica que se investiga es decir probabilidad en contra, de donde $q=1-p$, en este caso $q=1-0.5=0.5$

$e=$ representa el error muestral, que oscila entre $1 \%$ y un $5 \%$, queda a criterio del encuestador. En este caso se estimó en $5 \%$.

$$
\begin{gathered}
\text { Ecuación } 2 \\
\mathrm{n}=\frac{229 \times 1.96^{2} \times 0.5 \times 0.5}{(229-1) \times 0.05^{2}+1.96^{2} \times 0.5 \times 0.5}=144 \text { viviendas }
\end{gathered}
$$

El total de la muestra seleccionada para aplicar las encuestas fue de 715 viviendas para las

\begin{tabular}{|c|c|c|}
\hline Necesidad Básica & Contempla & Umbrales \\
\hline $\begin{array}{l}\text { Acceso a } \\
\text { Educación Básica }\end{array}$ & $\begin{array}{l}\text { Asistencia a escuelas de educación básica. } \\
\text { Hogares con niños en edad escolar que no } \\
\text { asiste a la escuela. }\end{array}$ & $\begin{array}{l}\text { Si al menos uno de sus integrantes } \\
\text { niño, o niña de edad entre } 7 \text { y } 12 \\
\text { años no asiste a ningún centro } \\
\text { educativo. }\end{array}$ \\
\hline $\begin{array}{l}\text { Acceso a Vivienda } \\
\text { Adecuada }\end{array}$ & $\begin{array}{l}\text { Calidad de la vivienda } \\
\text { Tipo de material de construcción en pisos, } \\
\text { paredes y techos. } \\
\frac{\text { Hacinamiento }}{\text { Hogares donde duermen muchas personas }} \\
\text { por habitación. } \\
\text { Espacio apropiado para cocinar } \\
\text { Asociado al espacio disponible, vinculado a } \\
\text { una actividad particular (cocinar) así como a } \\
\text { la calidad de ese espacio. }\end{array}$ & $\begin{array}{l}\underline{\text { Calidad de vivienda }} \\
\text { Si los materiales de las paredes y } \\
\text { techos son de desecho y piso de } \\
\text { tierra, la carencia es crítica. } \\
\frac{\text { Hacinamiento }}{\text { Si la cantidad de personas por }} \\
\text { habitación es más de tres. } \\
\frac{\text { Cocina }}{\text { Todo hogar que no disponga de un }} \\
\text { lugar apropiado para cocinar con } \\
\text { lavaplatos y grifo. }\end{array}$ \\
\hline $\begin{array}{l}\text { Disponibilidad de } \\
\text { Agua y Acceso } \\
\text { a Servicios } \\
\text { Sanitarios Básicos }\end{array}$ & $\begin{array}{l}\text { Abastecimiento de agua de buena calidad } \\
\text { en cantidades suficientes para satisfacer las } \\
\text { necesidades de alimentación e higiene y se } \\
\text { mide a partir de la potabilidad y la forma en } \\
\text { que ésta se suministra a la vivienda. } \\
\text { Acceso a servicios sanitarios contempla la } \\
\text { disponibilidad de servicio higiénico, es decir, } \\
\text { baño aislado con sistema de evacuación, } \\
\text { está asociado también a cuestiones de } \\
\text { salubridad y un sistema de eliminación de } \\
\text { aguas servidas. }\end{array}$ & $\begin{array}{l}\text { Abastecimiento de agua } \\
\text { Si ésta no llega por tuberías dentro } \\
\text { de la vivienda o si, en caso de } \\
\text { que lo haga, el origen no sea red } \\
\text { general ni pozo protegido. } \\
\text { Servicios sanitarios } \\
\text { Si la evacuación no se hace a } \\
\text { través de la red cloacal, o pozo } \\
\text { séptico, y si se comparte el baño } \\
\text { con otros hogares. }\end{array}$ \\
\hline
\end{tabular}
6 comunidades, aplicando una encuesta por cada habitante de la vivienda.

En la Tabla 1 se presentan los indicadores asociados a las necesidades básicas, y los umbrales seleccionados en esta investigación. 
(Continuación)

\begin{tabular}{lll} 
Necesidad Básica & Contempla & Umbrales \\
\hline Capacidad & Acceso a recursos económicos. & Recursos económicos. \\
\cline { 2 - 3 } Económica & Contempla el nivel educacional del jefe del & Si los jefes declararon como \\
& hogar y el número de personas a su cargo. & $\begin{array}{l}\text { máxima escolaridad segundo } \\
\text { grado de educación primaria y } \\
\text { mantienen a más de tres personas. }\end{array}$ \\
& & m
\end{tabular}

Tabla 1. Indicadores asociados a las necesidades básicas

Fuente: Autores, 2022, adaptado de CEPAL/PNUD (1989)

La metodología empleada se fundamentó en la implementación de reuniones, entrevistas y encuestas. Se aplicaron 715 encuestas en total entre las 6 comunidades. Cada familia seleccionó la persona que representaría del grupo familiar y respondería la encuesta, que fue aplicada por los grupos de trabajo que conforman esta investigación: Universidad de Carabobo (representada por estudiantes y docentes del CIHAM-UC y Bomberos Universitarios), Protección Civil Carabobo, Protección Civil Naguanagua (Farias et al., 2020). Las comunidades del Municipio Naguanagua seleccionados para el trabajo de campo desarrollado en el lapso 2019-2020 fueron: a) Brisas del Café, b) Las Adjuntas, c) Carialinda I, d) Carialinda II, e) Carialinda III, y f) Montesión.
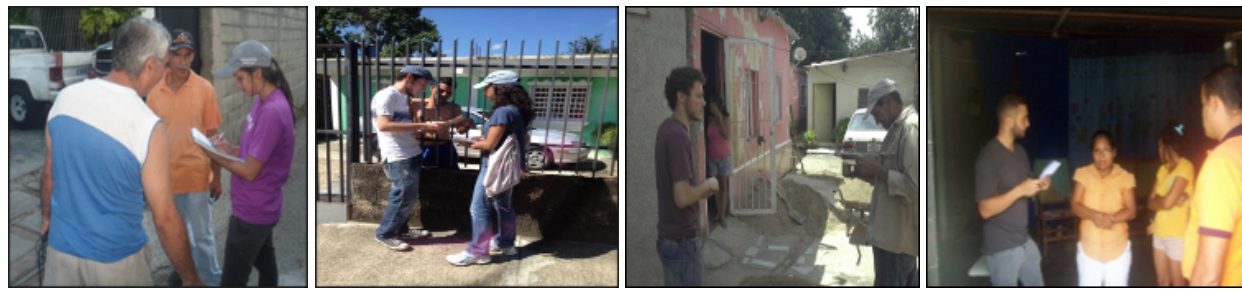

Figura 1. Aplicación de encuestas a los miembros de las diferentes comunidades

Fuente: Autores, 2019

\section{Identificación del escenario de riesgo de desastres}

Esta fase involucró las siguientes actividades:

a) Análisis de los antecedentes del escenario de desastres en el Municipio; los daños y pérdidas originados; descripción de los fenómenos amenazantes; posibles causas de los fenómenos amenazantes; determinación de factores que favorecen la ocurrencia de los desastres. Estas actividades se realizaron a través de búsqueda de material bibliográfico, de revisión de antecedentes de desastres reportados por Protección Civil, así como trabajo de campo, entrevistas a 18 líderes comunitarios que figuraban como representantes de las comunidades y 715 encuestas aplicadas a un representante de cada familia, mediante lo cual se recolectó la información necesaria.

b) Identificación del escenario según el criterio amenazante. Esta actividad incluyó la caracterización biofísica del Municipio; identificación de sus cuerpos de agua; condiciones climáticas; relieve; crecimiento urbano; geología y uso del suelo; actividades económicas; infraestructura vial; la modelación y simulación de las variables del balance hídrico y de las inundaciones que son herramientas tecnológicas que permitan distinguir las causas probables de los daños que ocasionan en la cuenca y así poder documentar los factores claves implicados en dichos daños (Farías et al., 2020b).

La metodología implementada incluyó: i) investigación documental; ii) consultas a representantes de Protección Civil Carabobo y a personal del cuerpo de Bomberos Universitarios, quienes han atendido a esas comunidades en casos de desastres; iii) aplicación de 715 encuestas a un miembro de cada una de las familias seleccionadas de las comunidades, donde se plantearon las siguientes interrogantes: ¿Indique cuál es el fenómeno natural que ocurre con más frecuencia en su comunidad?, ¿Conoce los riesgos a los que está expuesta su comunidad?, ¿Considera Ud. que su comunidad está preparada para reaccionar ante la ocurrencia de un desastre?, ¿Qué tan a menudo la comunidad se ve afectada por desastres y amenazas?, ¿Es la incidencia cada vez mayor la comunidad?, ¿Cuáles son los motivos principales de la vulnerabilidad en la comunidad? La información recabada fue digitalizada empleando la tecnología de los sistemas de información geográfica (SIG), que es empleada para diagnosticar sitios de posibles desbordes de cuerpos de agua y planificar acciones para mitigar la ocurrencia de inundaciones, a la vez que facilitan 
la caracterización de la distribución espacial de los asentamientos humanos dentro de las zonas urbanas y rurales (Farías et al., 2018) y la posición relativa respecto de elementos naturales (ríos, lagos, montañas, entre otros). La base de datos fue organizada empleando el software ArcGis 10.0, para realizar los mapas de zonificación de amenazas y de riesgos de inundación, sismos e incendios.

\section{PRESENTACIÓN DE RESULTADOS}

La información demográfica recabada fue organizada y se presenta en la Tabla 2.

\begin{tabular}{llllll} 
Comunidad & Viviendas & Habitantes & $\begin{array}{l}\text { Adolescentes y Adultos } \\
\text { (13 a 64 años) }\end{array}$ & $\begin{array}{l}\text { Niños } \\
\text { (7 a 12 años) }\end{array}$ & $\begin{array}{l}\text { Adultos Mayores } \\
\text { (> 65 años) }\end{array}$ \\
\hline Las Adjuntas & 46 & 368 & $60 \%(221)$ & $31 \%(114)$ & $9 \%(33)$ \\
\hline Colinas de Girardot I & 229 & 1100 & $70 \%(770)$ & $25 \%(275)$ & $5 \%(55)$ \\
\hline Colinas de Girardot II & 215 & 900 & $55 \%(495)$ & $35 \%(315)$ & $10 \%(90)$ \\
\hline Colinas de Girardot III & 545 & 1900 & $60 \%(1140)$ & $30 \%(570)$ & $10 \%(190)$ \\
\hline Brisas del Café & 100 & 600 & $70 \%(420)$ & $25 \%(150)$ & $5 \%(30)$ \\
\hline Montesión & 106 & 560 & $65 \%(364)$ & $25 \%(140)$ & $10 \%(56)$ \\
\hline Total & 1241 & 5428 & 3410 & 1564 & 454 \\
\hline Media & 206 & 904 & $63 \%$ & $24 \%$ & $8 \%$ \\
\hline
\end{tabular}

Tabla 2. Datos demográficos de las comunidades encuestadas Fuente: Autores, 2022

Los resultados de las encuestas se procesaron para obtener la información por comunidad que permitiera estimar el nivel de pobreza de cada una, presentando finalmente un gráfico por cada indicador para las 6 comunidades.

\section{Acceso a educación básica}

Con respecto a este indicador se agruparon primero las personas por grupos de edades, para poder discriminar el grupo de niños en edad comprendida entre 7 y 12 años que no asisten a las escuelas.

(a)

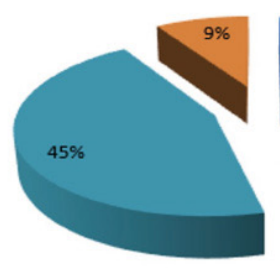

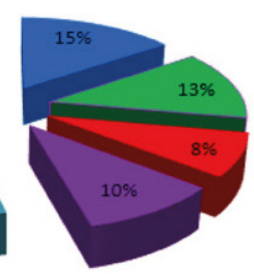

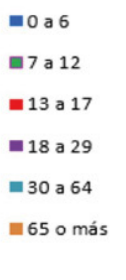

(b)

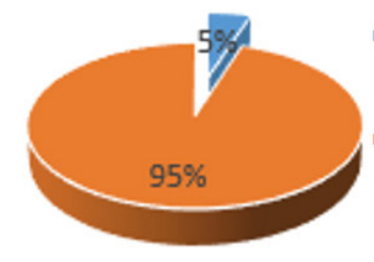

- NO ASISTE COLEGIO

SI ASISTE COLEGIO

Figura 2. Distribución de la población por edades en años, y \% de asistencia a escuelas Fuente: Autores, 2022

La Figura 2a representa la distribución en edades de las poblaciones de las seis comunidades, donde el $13 \%$ corresponde a niños en edad escolar (entre 7 y 12 años), la Figura 2b presenta el porcentaje de niños entre 7 y 12 años de edad que no asisten a las escuelas, y corresponde al $5 \%$ de la muestra. Es decir, esta necesidad básica está cubierta en casi todas las comunidades.

\section{Acceso a vivienda adecuada}

Con respecto a esta necesidad básica se evaluaron tres indicadores:

\section{Tipo de material de construcción de paredes techos y pisos}

Para estimar este indicador se empleó la técnica de la observación directa, para inspeccionar la vivienda y evaluar material de construcción de techos y paredes, y recubrimiento del piso. La Figura 3 muestra diferentes escenas donde se visualiza el tipo de material de fabricación de algunas de las viviendas visitadas. Los resultados de este indicador se muestran en la Figura 4a. De la muestra seleccionada, el $75 \%$ habita en viviendas con materiales de paredes, techos y pisos en buenas condiciones, mientras que el $25 \%$ restante tiene las viviendas construidas con láminas de zinc, maderas y material de desecho, y poseen pisos de tierra compactados, o apenas una lechada de cemento. 

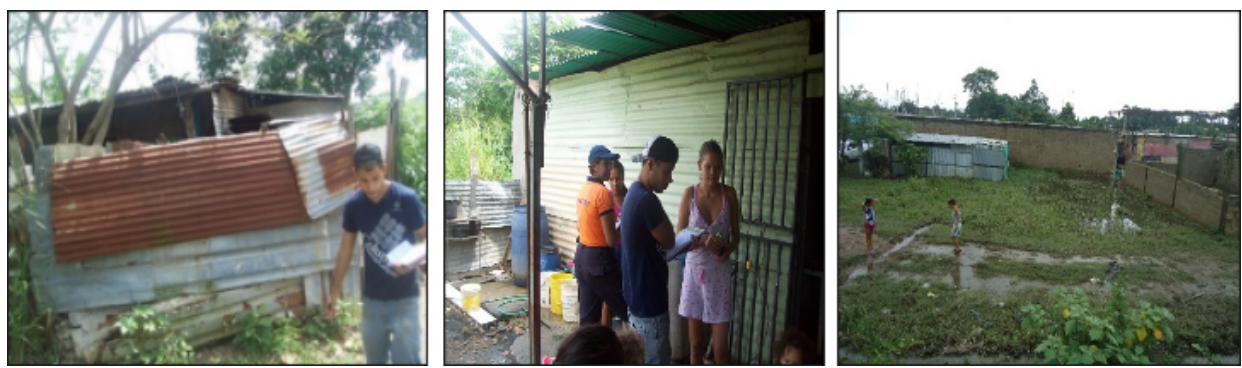

Figura 3. Muestra de material de construcción de algunas viviendas Fuente: Autores, 2019

\section{Hacinamiento}

El grado de hacinamiento de los habitantes de las viviendas se determinó de acuerdo a las respuestas obtenidas de las entrevistas. Los resultados se presentan en la Figura 4b, reflejando que el $90 \%$ de las familias no poseen condiciones para tener a sus integrantes en habitaciones separadas, y comparten 3 o más personas la misma habitación.

\section{Área de cocina}

El sitio para preparar los alimentos en un alto porcentaje está separado de las habitaciones, pero lo comparten como sala y comedor en un solo ambiente. La Figura 4c se presentan los resultados obtenidos, donde un $30 \%$ de la muestra no posee la cocina adecuada según el umbral de este indicador, la cocina no está separada de las habitaciones, no posee las condiciones mínimas para preparar los alimentos, no tienen lavaplatos ni llaves de agua.

(a)

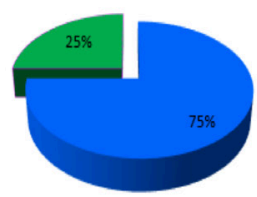

(b)

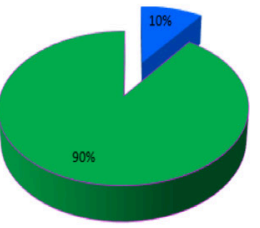

(c)

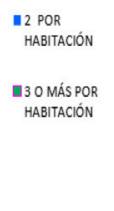

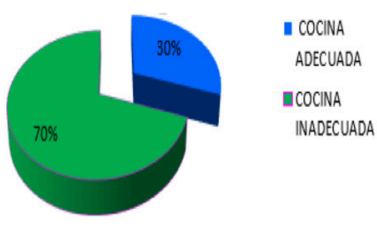

Figura 4. Representación de los indicadores para evaluar el acceso a vivienda adecuada Fuente: Autores, 2022

\section{Disponibilidad de agua y acceso a servicios sanitarios básicos}

El acceso a servicios básicos como la disponibilidad de acueductos y cloacas a las viviendas de las comunidades encuestadas se determinó de acuerdo a las respuestas suministradas en las entrevistas. La Figura 5 a muestra que el $90 \%$ de las viviendas poseen acceso a la red de agua del Instituto encargado de proveerla, sin embargo, declaran tener muchos meses sin recibirla. En este caso se sumó como una NBI por que no cuentan con el servicio. Con respecto a la conexión a cloacas se presenta en la Figura $5 \mathrm{~b}$ que el $85 \%$ de las viviendas cuentan con este servicio, pero un $15 \%$ descarga a ríos, quebradas o canales adyacentes a sus viviendas.

(a)

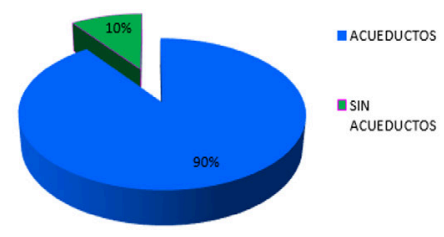

(b)

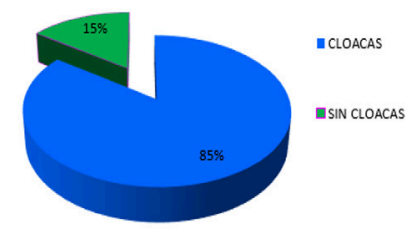

Figura 5. Representación de los indicadores para evaluar la disponibilidad de agua y servicios sanitarios Fuente: Autores, 2022

\section{Capacidad económica}

Para determinar la cobertura de esta necesidad básica se estimaron dos indicadores el grado de educación del jefe de familia, y el número de personas que dependen de sus ingresos. En un alto porcentaje de los entrevistados se observó que en un $90 \%$ el hombre era el que trabajaba fuera del hogar, la mujer se dedica al trabajo doméstico y cuidar a los hijos, que como mínimo tienen tres 
por familia. La Figura 6a presenta los resultados del grado de instrucción de los integrantes de las familias, donde un $35 \%$ no terminaron la escuela primaria, un $26 \%$ no culminaron la secundaria, pero si hay un grupo de personas que representan el 10\% con secundaria completa, un $8 \%$ con grados de técnicos y un $1 \%$ de profesionales universitarios. Con respecto al grado de dependencia del jefe de familia el $93 \%$ de los encuestados dependen del padre o madre quien cumpla ese rol; es decir cada jefe de hogar en promedio mantiene a 4 personas.

(a)

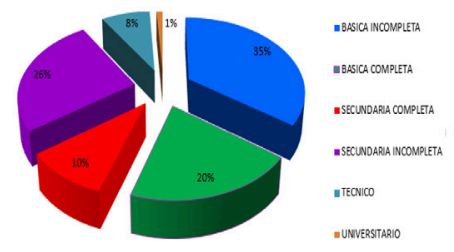

(b)

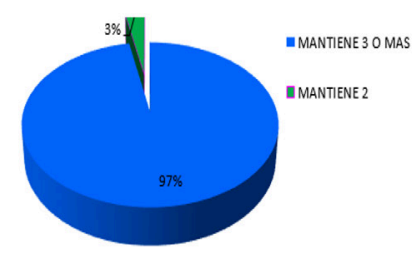

Figura 6. Representación de los indicadores para evaluar grado de instrucción de los miembros de las familias Fuente: Autores, 2022

En la Tabla 3 se presentan los resultados correspondientes a los indicadores asociados a la medición de necesidades básicas insatisfechas (NBI), y necesidades básicas satisfechas (NBS) para las comunidades seleccionadas en esta investigación.

\begin{tabular}{llllll} 
Comunidad & $\begin{array}{l}\text { Muestra } \\
\text { Hogares/ } \\
\text { Habitantes }\end{array}$ & $\begin{array}{l}\text { Educación } \\
\text { Personas } \\
\text { NBS/NBI }\end{array}$ & $\begin{array}{l}\text { Vivienda } \\
\text { Hogares NBS/ } \\
\text { NBI }\end{array}$ & $\begin{array}{l}\text { Servicios } \\
\text { Hogares NBSI } \\
\text { NBI }\end{array}$ & $\begin{array}{l}\text { Recursos } \\
\text { personas } \\
\text { NBS/NBI }\end{array}$ \\
\hline Las Adjuntas & $46 / 368$ & $108 / 6$ & $4 / 42$ & $7 / 39$ & $14 / 354$ \\
\hline Colinas de Girardot I & $144 / 432$ & $259 / 16$ & $12 / 132$ & $33 / 111$ & $16 / 416$ \\
\hline Colinas de Girardot II & $138 / 276$ & $299 / 10$ & $13 / 125$ & $28 / 110$ & $17 / 259$ \\
\hline Colinas de Girardot III & $225 / 675$ & $512 / 29$ & $26 / 199$ & $15 / 210$ & $41 / 634$ \\
\hline Brisas del Café & $80 / 240$ & $142 / 8$ & $8 / 72$ & $12 / 68$ & $13 / 227$ \\
\hline Montesión & $82 / 246$ & $133 / 8$ & $9 / 73$ & $12 / 70$ & $14 / 230$ \\
\hline Total & $715 / 2237$ & $1453 / 77$ & $72 / 643$ & $107 / 608$ & $115 / 2121$ \\
\hline
\end{tabular}

Tabla 3. Distribución del grado de satisfacción de necesidades básicas Fuente: Autores, 2022

De los 4 indicadores medidos solo se cumple con acceso a la educación de los niños en edad escolar. Lo que convierte a los habitantes de estas comunidades en personas pobres de acuerdo a la metodología del NBI, existiendo incluso familias con todas las necesidades insatisfechas, que los coloca en el grupo de venezolanos con pobreza extrema.

\section{Identificación de escenario de riesgo de desastres}

Análisis de los antecedentes de los escenarios de riesgos de desastres del estado

\section{Carabobo}

El estado Carabobo se ubica sobre un eje de numerosas fallas activas y de zonas de inestabilidad en la franja centro-norte-costera venezolana la cual está propensa a sufrir movimientos telúricos de gran magnitud, con la evidencia de haber sido epicentro de varios sismos. A lo largo del eje se asienta una alta densidad poblacional, que, sumada al tipo de construcciones, inciden en el riesgo sísmico tipificado para el estado, el cual está clasificado entre 4 y 5 en las zonas vulnerables que representan de medio a alto índice de peligrosidad sísmica, y posibles deformaciones en la superficie terrestre como efectos secundarios (Venezuela, 2001).

A pesar de que Venezuela se encuentra en el extremo sur de la zona donde los huracanes son frecuentes, en forma ocasional las costas del estado están expuestas, y en la práctica pueden ser afectadas por huracanes que se formen en los $10^{\circ}$ de latitud Norte (aunque la mayoría se producen sobre los $12^{\circ}$ ) (Decarli, 2010). Por lo tanto, las zonas costeras pueden ser afectadas por tormentas tropicales o huracanes, y representan alta vulnerabilidad para la población ubicada en las zonas bajas. También los movimientos de masas constituyen alta vulnerabilidad en las 
zonas montañosas inestables, donde representan un peligro común para las viviendas precarias y para la vialidad. Se localizan principalmente en los municipios San Joaquín, Guacara, San Diego, Naguanagua y en la zona sureste de Puerto Cabello (Oficina de Investigaciones Geográficas, 2003), y, por último, el riesgo por inundaciones se presenta con mayor frecuencia en las áreas con pendientes menores del $3 \%$, localizándose en los fondos de valle (Arismendi, 2007).

\section{Escenario de riesgo del municipio Naguanagua}

El municipio Naguanagua presenta dos riesgos importantes: sismos e inundaciones. Su ubicación en una zona sísmica catalogada de nivel 5 representa un riesgo elevado, influenciada por las fallas la Guacamaya, las Trincheras y la Victoria (Venezuela, 2001), lo convierte en una zona de alto riesgo sísmico.

Con respecto al riesgo de inundación existen corrientes fluviales del municipio como: el río Cabriales que recorre la ciudad de Valencia de norte a sur hasta llegar a su desembocadura en el Lago de Valencia; río El Retobo se extiende por todo el norte del municipio hasta su confluencia con el río Cabriales; río Aguas Calientes y muchas quebradas que forman parte de la red hídrica del municipio y atraviesan estas comunidades, presentando además exceso de malezas en sus cauce, erosión del fondo de los ríos, y grandes cantidades de basura que contribuyen a su desborde en épocas de lluvia. Existen además infraestructuras muy vulnerables como las viviendas construidas con material de desecho, ubicadas dentro de las planicies de inundación, ausencia de drenajes y de servicios públicos.

Entre las posibles causas que favorecen la ocurrencia de desastres en el municipio están: los efectos del cambio climático; destrucción de la capa vegetal por factores antrópicos y naturales (sequía, incendios); disminución de la infiltración; incremento de la escorrentía y erosión masiva; ocurrencia de sismos desestabilizadores de masas de materiales; la topografía pasa de suavemente inclinada con una pendiente promedio del 3\% al 5\% (Arismendi, 2007); la constitución de los suelos donde prevalecen hacia el valle suelos de textura predominante franco arcillo-arenosa/ gravosa (por aportes laterales), generalmente bien drenados (Farias et al., 2018); la tectónica local y regional; así como la actividad antrópica representada en la deposición de sólidos en los cauces; el mal manejo de aguas negras; canalizaciones de aguas negras y de lluvia dispuestas a los ríos; entre otros.

\section{Identificación del escenario según el criterio amenazante}

La Figura 7a muestra la distribución de los eventos que han ocurrido con más frecuencia en las comunidades. Obsérvese que el $77 \%$ de la población coincide que las inundaciones son el fenómeno natural que ha ocurrido con más frecuencia en sus comunidades, mientras que $5 \%$ opina que han sido los vientos muy fuertes descritos por ellos como huracanados. El $12 \%$ reporta la ocurrencia de sismos, otro $5 \%$ ha sufrido de deslaves en su comunidad y un $1 \%$ se ha visto afectado por incendios forestales, precisamente las familias con viviendas muy cercanas a zonas verdes que en época de sequía se incendian frecuentemente.

La Figura $7 b$ muestra la respuesta a la pregunta si ellos creen que viven en situación de riesgos, el 90\% reconoce vivir expuesto a riesgos de desastres. Y, por último, la Figura 7c muestra la respuesta a la pregunta si ellos creen estar preparados para actuar ante un desastre: el $99 \%$ reconoce no tener preparación para comportarse ante este tipo de eventos.

(a)

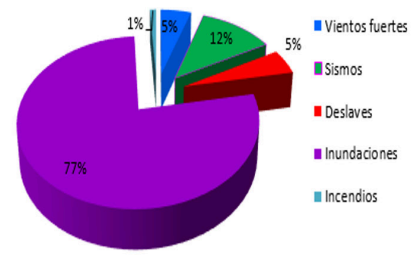

(b)

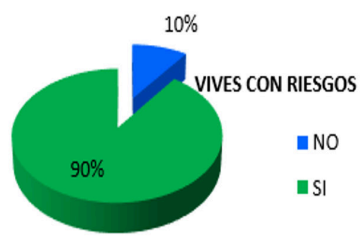

(c)

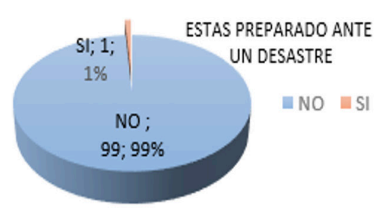

Figura 7. Representación de los indicadores para evaluar el escenario según la amenaza Fuente: Autores, 2022

En todas estas comunidades existe una amenaza o peligro latente de que un evento de origen natural, como las lluvias causantes de las inundaciones, se presente con una severidad suficiente 
para causar pérdidas de vidas, lesiones u otros impactos en la salud, así como también daños y pérdidas en los bienes, la infraestructura, los medios de sustento, la prestación de servicios y los recursos ambientales (Farías et al., 2020a), como se puede observar en la Figura 8 donde se muestran escenas de la ubicación de las viviendas a las márgenes de los ríos. Esto confirma la premisa que los desastres no son naturales, sino que el factor humano siempre incide, que la vulnerabilidad es una construcción social, así como las amenazas son una modalidad de desarrollo y formas de producción (Lavell, 2004).
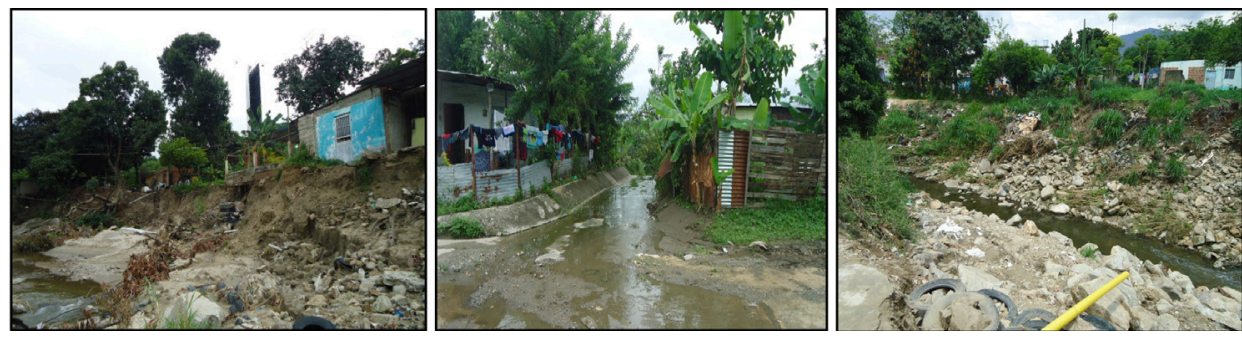

Figura 8. Escenas de ubicación de las viviendas de las comunidades a orillas de los ríos Fuente: Autores, 2022

(a)

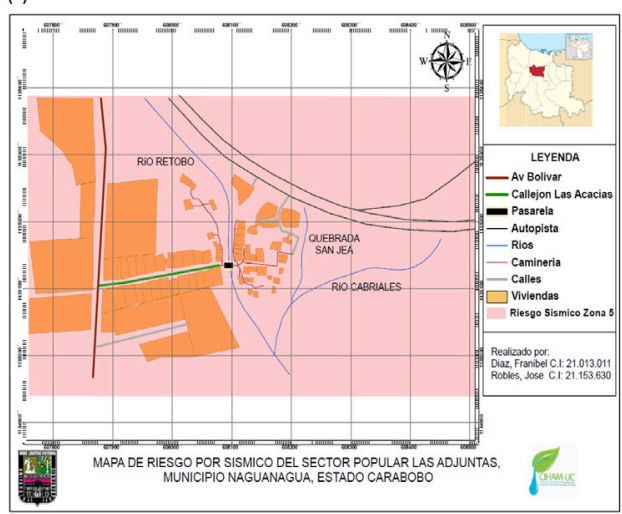

(b)

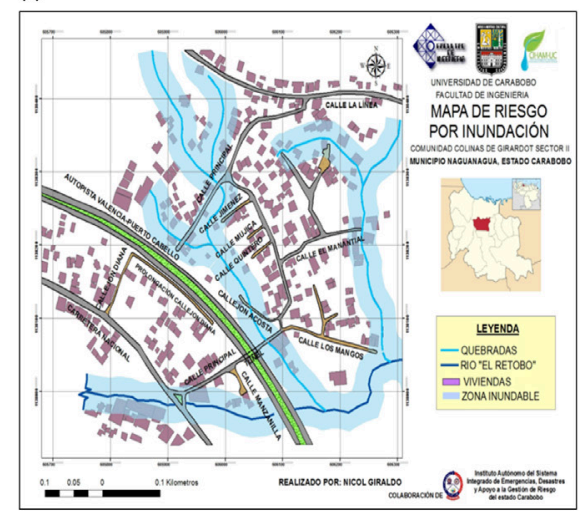

Figura 9: (a) Mapa de riesgo sísmico en el sector Las Adjuntas; b) Mapa de riesgo de inundación de Colinas de Girardot II Fuente: Autores, 2022

Entre de las actividades realizadas en esta fase están la construcción de los mapas de riesgos de inundación y sismos de cada una de las comunidades. Los mapas son una herramienta visual recomendados para comunicar las amenazas y riesgos que existen en un área (Abhas, 2012). Como una muestra se presenta en la Figura 9a el mapa de riesgo de sismos de la comunidad Las Adjuntas, toda la comunidad está encerrada en un polígono color rosa porque toda está expuesta a sismos. La Figura 9b muestra el mapa de inundación de la comunidad Colinas de Girardot sector II, donde puede observarse que las viviendas representadas en color morado están ubicadas en las márgenes de ríos y quebradas.
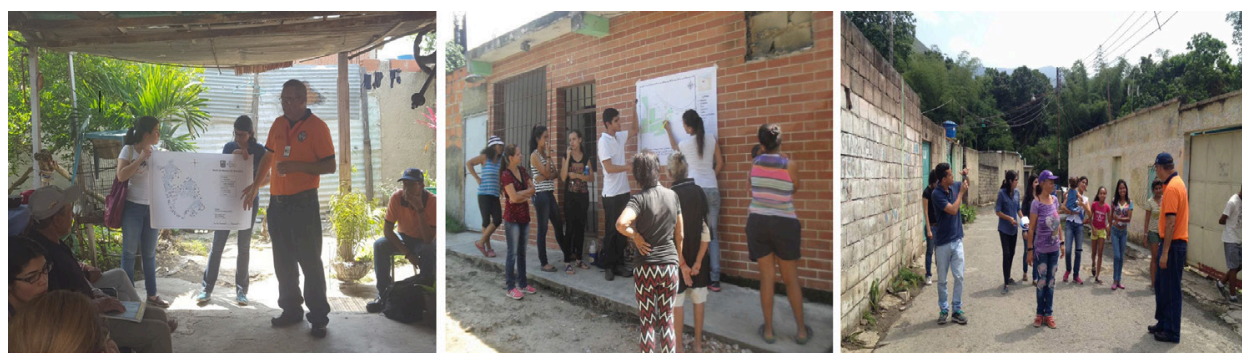

Figura 10. Escenas de difusión de los diferentes mapas obtenidos en la investigación Fuente: Autores, 2022

Además, se realizaron mapas de rutas de evacuación donde se señalan las calles, los sitios aptos para refugios y las zonas seguras de acuerdo a la información suministrada por las comunidades. Toda la información generada fue compartida con las personas de las comunidades como puede observarse en las Figura 10, donde también se aprecia cuando se recorre una ruta de evacuación hacia una zona segura con el personal de Protección Civil. 


\section{CONCLUSIONES}

De lo antes expuesto se pudo concluir que la pobreza se expresa en las condiciones de vida precarias donde prevalecen las condiciones de viviendas inadecuadas y la inexistencia de servicios públicos. Las comunidades asentadas en zonas inundables, o de alto riesgo sísmico, resultan directamente afectadas. Además, un alto porcentaje de ellas presenta desnutrición, vulnerabilidad a las enfermedades e insuficiencia de servicios de salud y abastecimiento de medicamentos.

Con respecto a los habitantes de las comunidades de este estudio, son personas de escasos recursos económicos lo cual influye directamente en la capacidad de respuesta y recuperación ante situaciones de desastres. De las respuestas recibidas por los habitantes seleccionados en la muestra de la investigación, todos plantean vivir en esas zonas a orillas de los ríos por no tener otras alternativas, su condición económica no les permite construir en otras zonas más seguras. Pero esta situación se puede extrapolar al resto de los habitantes al observar las condiciones de vida que persisten en esas comunidades. De lo expuesto por ellos mismos, estas comunidades no disponen de ayuda por parte de los organismos encargados de brindar tanto apoyo institucional como técnico. Existe poca o ninguna organización comunitaria que les permita estar bien preparados para el manejo del riesgo, la mitigación y la prevención ante los desastres asociados a fenómenos naturales (Farias et al., 2017).

Con los resultados se confirmaron varios aspectos de las comunidades que participaron en este proyecto:

a. Todas califican de pobres bajo el criterio que es pobre aquella persona que no logra satisfacer sus necesidades básicas de alimentación, vivienda, vestido y educación; al aplicar los indicadores todas poseen más de una necesidad básica insatisfecha, encontrándose que la comunidad Colinas de Girardot III está en el rango de pobreza extrema al presentar 3 índices de insatisfacción de los 4 medidos. Es donde mayor número de personas comparten la misma habitación, y poseen mayor problema de aducción de aguas blancas. En segundo lugar, está Colinas de Girardot II y le sigue Colinas de Girardot I;

b. En general todas las comunidades presentan alta vulnerabilidad frente a los desastres, como se pudo verificar con la construcción de los mapas de riesgos elaborados con apoyo de la comunidad y que además fueron compartidos con ellos aprovechando para ser verificados. En el caso de rutas de evacuación y selección de zonas seguras para cada uno de los riesgos existentes se recorrieron las rutas con las personas de cada comunidad, e incluso se realizaron simulacros de evacuación;

c. Presentan una sobreexposición a eventos frecuentes y de baja intensidad, como las inundaciones recurrentes, ya que en todas las comunidades existen ríos y quebradas, donde estas familias han construido sus viviendas, aun a sabiendas que están expuestos a desastres, como lo demostraron los resultados de las encuestas que reflejaron un $90 \%$ de aceptación del conocimiento del riesgo a inundaciones, y que el $99 \%$ no está preparado para actuar en caso de desastres.

Todo lo expuesto permite concluir que los pobres sufren mayor afectación por los desastres, ya que su pérdida es proporcional a su riqueza, y sus activos conformados por viviendas inadecuadas y medios de vida caracterizados por ausencia de servicios básicos los convierte en personas más vulnerables.

Otro aspecto destacado en esta investigación es la posición de Venezuela frente al cumplimiento de los ODS: no se reportan avances de su gestión desde 2016, ni se han definido mecanismos de información de avances, lo cual es preocupante porque demuestra la inactividad para cumplir al menos con disminuir la pobreza extrema que a su vez favorecería la disminución de la vulnerabilidad de estas comunidades a los riesgos hidrometeorológicos.

Como alternativa para apoyar a las comunidades se plantea: elaboración de políticas y planes de gestión de riesgos que reduzcan la vulnerabilidad, que definitivamente está influenciada por las características socioeconómicas de la población ubicada en las planicies de inundación (Farías et al., 2020c); brindar el potencial necesario para construir procesos de desarrollo sustentable a las comunidades más pobres, que viven las consecuencias de los eventos climáticos extremos. 


\section{REFERENCIAS}

Jha, Abhas K.; Bloch, R.; Lamond, J. (2012). Ciudades e Inundaciones. Guía para la Gestión Integrada del Riesgo de Inundaciones en Ciudades en el Siglo 21 Resumen para hacedores de políticas. Incluye estudios de caso y ejemplos de la región latinoamericana. Banco Mundial. https://reliefweb.int/sites/ reliefweb.int/files/resources/Situation_Report_156.pdf

Acuña, V.; Valdivieso, S.; Juzam, L. (2021). Dignificando la Gestión de Riesgo de Desastres: liderazgos femeninos y estrategias comunitarias en el Campamento Dignidad, Santiago de Chile. Revista de Estudios Latinoamericanos sobre Reducción del Riesgo de Desastres REDER, 5(2), 91-106. http:// revistareder.com/handle-0719-8477-2021-096

Aponte, C.; Romero, E.; Santa, L.F. (2015). Análisis de datos espaciales del Îndice de Necesidades Básicas Insatisfechas en la Región Andina. Perspectiva Geográfica, 20(2), 391-418. http://www.scielo.org.co/ pdf/pgeo/v2onz/v2onzao8.pdf

Arias, F. (2012). El proyecto de investigación. Introducción a la metodología (6ta Ed.). Editorial Episteme. https://es.slideshare.net/juancarlos777/el-proyecto-de-investigacion-fidias-arias-2012-6a-edicion

Arismendi, J. (2007). Presentación geográfica de las formas de relieve. Geo Venezuela (Tomo 2). Fundación Empresas Polar. https://bibliofep.fundacionempresaspolar.org/publicaciones/ colecciones/geovenezuela/

Arrillaga, H.; Grand, M.; Busso, G. (2009). Vulnerabilidad, riesgo y desastres. Sus relaciones de causalidad con la exclusión social en el territorio urbano santafesino. En: Herzer, H.; Arrillaga, H. (Ed.), La construcción social del riesgo y el desastre en el aglomerado Santa Fe. Universidad Nacional del Litoral, 2009, p.59-104.

Battinson, D. (2007). Refinando el Enfoque de la Necesidad Básica: Un análisis multidimensional de la pobreza en América Latina. OPHI Working Papers, 17. https://www.academia.edu/19256176/ Refining_the_Basic_Needs_Approach_A_multidimensional_analysis_of_poverty_in_Latin_America

Blaikie, P. (1996), Vulnerabilidad, el entorno social, político y económico de los desastres. Red de Estudios Sociales en Prevención de Desastres en America Latina (LA RED). https://www. desenredando.org/public/libros/1996/vesped/

Blaikie, P.; Cannon, T.; Davies, I.; Wisner, B. (1996). Vulnerabilidad. El entorno social, político y económico de los desastres. La Red de Estudios Sociales en Prevención de Desastres en América Latina (LA RED). http://cidbimena.desastres.hn/pdf/spa/doc840o/doc840o-indice.pdf

Calvo, J.; Giraldez, C. (2000). Las necesidades básicas insatisfechas en Montevideo de acuerdo al Censo de 1996. Documento de Trabajo N$^{\circ} 44$. Unidad Multidisciplinaria, FCS, UdelaR. http://fcea.edu.uy/ Jornadas_Academicas/2013/file/ECONOMIA/Ponencia_NBI_Calvo_Carrasco.pdf

Calvo, J. (2014). Atlas sociodemográfico y de la desigualdad del Uruguay: las Necesidades Básicas Insatisfechas a partir de los Censos 2011, VI Congreso de la Asociación Latinoamericana de Población (ALAP). Dinámica de población y desarrollo sostenible con equidad. Lima, Perú, agosto 2014. https:// uruguay.unfpa.org/sites/default/files/pub-pdf/122_file1.pdf

CRED (Centro para la Investigación de la Epidemiología de los Desastres). (2013). EM-DAT: La Base de Datos Internacional de Desastres: Tendencias de desastres. Universidad Católica de Lovaina. www. emdat.be/disaster-trends

CEPAL. (2001). Enfoques para la medición de la pobreza. Breve revisión de la literatura. División de Estadística y Proyecciones Económicas, CEPAL. https://www.cepal.org/es/ publicaciones/4740-enfoques-la-medicion-la-pobreza-breve-revision-la-literatura

CEPAL. (2014). Manual para la evaluación de desastres. CEPAL https://www.cepal.org/es/ publicaciones/35894-manual-la-evaluacion-desastres

CEPAL. (2016). La matriz de la desigualdad social en América Latina. CEPAL. https://www.cepal.org/ sites/default/files/events/files/matriz_de_la_desigualdad.pdf

CEPAL. (2018). Panorama social de América Latina 2018. CEPAL. https://www.cepal.org/es/ publicaciones/44395-panorama-social-america-latina-2018\#: :text=El\%20Panorama\%2oSocial\%20 2018\%2oanaliza,en\%2olos\%2omercados\%2ode\%2otrabajo

CEPAL. (2019). Informe de avance cuatrienal sobre el progreso y los desafíos regionales de la Agenda 2030 para el Desarrollo Sostenible en América Latina y el Caribe. CEPAL. https://news.un.org/es/ story/2018/09/1441532

Cutter, S.; Mitchell, J.; Scott, M. (200o). Revealing the vulnerability of people and places: a case study of Georgetown County, South Carolina. Annals of American Geographers, 9o(4), 713-737 
Cutter, S.; Boruff B.; W. Lynn, S. (2003). Social vulnerability to environmental hazards, Social Science Quarterly, 84(2). https://doi.org/10.1111/1540-6237.8402002

Cutter, S.; Emrich C.; Webb, J.; Morath D. (2009). Social vulnerability to climate variability hazards: a review of the literature", Final Report to Oxfam America. Hazards and Vulnerability Research Institute, University of South Carolina.

Decarli, F. (2010). Estado Carabobo. Geo Venezuela (Tomo 6). Fundación Empresas Polar. http:// docplayer.es/189113039-Fundacion-empresas-polar-caracas-geo.html

EIRD. (2009). Informe de evaluación global sobre la reducción del riesgo de desastres. Naciones Unidas. http://hdl.handle.net/20.500.11762/19779

Encovi. (2020). Encuesta Nacional de Condiciones de Vida 2019-2020. Venezuela. Encovi. https://www. proyectoencovi.com/

Farias, B.; Márquez, A.; Guevara, E.; Rey, D. (2018). Caracterización espacio-temporal del uso de la tierra en cuencas hidrográficas mediante técnicas geomáticas. Revista Ingeniería UC, 25(1).19-30. http:// servicio.bc.uc.edu.ve/ingenieria/revista/v25n1/vol25n12018.pdf

Farías, B.; Márquez A.; Guevara E; Romero, A. (2017). Una metodología para prevenir y afrontar el riesgo hidrológico en la zona norte del municipio Naguanagua estado Carabobo - Venezuela. Revista Encuentros Unellez,1(1),86-102. https://revistaencuentroscies.wordpress.com/2018/o6/10/ volumen-1-n-1-julio-diciembre-2017/

Farias, B.; Marquez, A.; Guevara, E.; Rey, D. (2020a). Plan de gestión sustentable de riesgo de inundación: Una propuesta desde Carabobo Venezuela. REDER Revista de Estudios Latinoamericanos sobre Reducción del Riesgo de Desastres. 4(1), 67-79. http://revistareder.com/ojs/ index.php/reder/article/view

Farias, B.; Marquez, A.; Guevara, E.; Rey, D. (2020b). Geostatistical modeling of surface water balance (SWB) under variable soil moisture conditions in the Pao river basin, Venezuela. DYNA. https://doi. org/10.15446/dyna.v87n213.80446

Farias, B.; Marquez, A.; Guevara, E.; Rey, D. (2020c). Method for flood risk estimation in a tropical basin. Water Supply Journal, 2o(2), 712-723. https://doi.org/10.2166/ws.2019.202

Feres, J.; Mancero, X. (2001). El método de las necesidades básicas insatisfechas (NBI) y sus aplicaciones en América Latina. Naciones Unidas \& División estadística y Proyecciones Económicas, CEPAL. https://www.cepal.org/es/ publicaciones/4784-metodo-necesidades-basicas-insatisfechas-nbi-sus-aplicaciones-america-latina

Fernández, T. (2012). Metodología preliminar para la medición multidimensional de la pobreza en Uruguay. En: Cortés, F. \& Mora, M. (Eds.), Metodología multidimensional de medición de la pobreza. El Colegio de México. https://www.coneval.org.mx/rw/resource/Metodologia_Medicion_ Multidimensional.pdf

Hallegatte, S.; Bangalore, M.; Bonzanigo, L.; Fay, M.; Kane, T.; Narloch, U.; Rozenberg, J.; Treguer, D.; Vogt-Schilb, A. (2015). Shock Waves: Managing the Impacts of Climate Change on Poverty. Climate Change and Development Series, World Bank. https://openknowledge.worldbank.org/ handle/10986/22787

Hallegatte, S.; Vogt-Schilb, A.; Bangalore, M.; Rozenberg, J. (2017). Indestructibles: Construyendo la resiliencia de los más pobres frente a desastres naturales. Banco Mundial. https://es.scribd.com/ document/331312718/Informe-Banco-Mundial

INE (Instituto Nacional de Estadísticas). (2011). Resumen de indicadores socioeconómicos. INE. http:// www.ine.gov.ve/documentos/SEN/menuSEN/pdf/subcomitesociales/Indicadores_Disponibles.pdf

Kim, N. (2012). How much more Exposed are the Poor to Natural Disasters? Global and Regional Measurement. Disasters, 36(3), 558-558. https://doi.org/10.1111/j.1467-7717.2011.01258.x

Klisberg, B; Sen A. (2009). Una mirada desde la ética del desarrollo a los principales problemas del mundo globalizado (6ta Edición). Editorial Temas. https://www.revistas.una.ac.cr/index.php/ri/ article/view/5172

Lavell, A. (2004). Antecedentes, formación y contribución al desarrollo de conceptos, estudios y la práctica en el tema de los riesgos y desastres en América Latina: 1980-2004. La Red de Estudios Sociales en Prevención de Desastres en América Latina (LA RED).

Lusseau, D.; Mancini, F. (2019). Income-Based Variation in Sustainable Development Goal Interaction Networks. Nature Sustainability, 2, 242-247. https://doi.org/10.1038/s41893-019-0231-4 
Maskrey, A. (1993). Los desastres no son naturales. La Red: Red de estudios sociales en prevención de desastres en América Latina (LA RED).

NU (Naciones Unidas). (2019). Informe Mundial sobre el Desarrollo Sostenible: El futuro es ahora: Ciencia para lograr el desarrollo sostenible. Naciones Unidas.

NU (Naciones Unidas). (2018). La Agenda 2030 y los Objetivos de Desarrollo Sostenible: una oportunidad para América Latina y el Caribe. Naciones Unidas.

Oficina de Investigaciones Geográficas (OIG). (2003). Influencia del eje económico Mariara, ValenciaPuerto Cabello en el patrón de distribución de la población del estado Carabobo, Caracas. OIG.

Phelean, M. (2006). La pobreza en Venezuela. Fundación Escuela de Gerencia Social, Ministerio del Poder Popular para la Planificación y Desarrollo.

PNUD (Programa de las Naciones Unidas para el Desarrollo). (2010). Informe de Desarrollo Humano (2010): Medición de pobreza y desarrollo en América Latina. PNUD. http://hdr.undp.org/sites/ default/files/rhdr-2010-rblac.pdf

PNUD (Programa de las Naciones Unidas para el Desarrollo). (2011). Informe sobre el Desarrollo Mundial 2011: Sostenibilidad y Equidad: Un mejor futuro para todos. PNUD. https://www.undp.org/ content/undp/es/home/librarypage/hdr/human_developmentreport2o11.html

PNUD (Programa de las Naciones Unidas para el Desarrollo). (2014). Informe sobre Desarrollo Humano 2014: Sostener el Progreso Humano: Reducir vulnerabilidades y construir resiliencia. PNUD. https:// www1.undp.org/content/undp/es/home/librarypage/hdr/2014-human-development-report.html

PNUD (Programa de las Naciones Unidas para el Desarrollo). (2019). Informe sobre Desarrollo Humano 2019 Más allá del ingreso, más allá de los promedios, más allá del presente: Desigualdades del desarrollo humano en el siglo XXI. PNUD. http://hdr.undp.org/sites/default/files/hdr_2019_ overview_-_spanish.pdf

PNUD (Programa de las Naciones Unidas para el Desarrollo). (2018). Cambiar, Mejorar, Actuar. PNUD. https://www1.undp.org/content/undp/es/home/librarypage/corporate/annual-report-2018.html

PNUD (Programa de las Naciones Unidas para el Desarrollo). (2020a). Panorama general: El desarrollo humano y el Antropoceno, Estructura del Informe sobre Desarrollo Humano 2020. PNUD. http://hdr. undp.org/sites/default/files/hdr_2020_overview_spanish.pdf

PNUD (Programa de las Naciones Unidas para el Desarrollo). (2020b). COVID-19 y Desarrollo Humano: Evaluando la Crisis, Visualizando la Recuperación. PNUD. http://hdr.undp.org/en/hdp-covid

PNUD (Programa de las Naciones Unidas para el Desarrollo); OPHI (Iniciativa de Pobreza y Desarrollo Humano de Oxford). (2020). Índice de Pobreza Multidimensional global 2020. Trazar caminos para salir de la pobreza multidimensional: Lograr los ODS. UNDP. http://hdr.undp.org/sites/default/ files/2020_mpi_report_es.pdf

Rincón S.; Berosca, I. (2014). Pobreza en Venezuela (2000-2010). Omnia, 20(2). 162-176. https://www. redalyc.org/pdf/737/73735396o11.pdf

Saad, L. (2019, 25 de marzo). Los estadounidenses están tan preocupados como siempre por el calentamiento global. Gallup. https://news.gallup.com/poll/248027/americans-concerned-everglobal-warming.aspx

SINERGIA. (2019). Red Venezolana de Organización de la Sociedad Civil. Los objetivos de desarrollo sostenible en Venezuela: Reporte de un país en riesgo 2019. SINERGIA. https://transparencia.org.ve/ sinergia-ods-en-venezuela-reporte-desde-un-pais-en-riesgo/

Scussolini, P.; Aerts, J.C.J.H.; Jongman, B.; Bouwer, L.M.; Winsemius, H.C.; De Moel, H.; Ward, P.J. (2016). FLOPROS: An Evolving Global Database of Flood Protection Standards. Natural Hazards and Earth System Sciences 16, 1049-1061. https://doi.org/10.5194/nhess-16-1049-2016

Suárez, M; Tapia, F. (2012). Interaprendizaje de Estadística Básica. Universidad Técnica del Norte. Facultad de Ciencias Administrativas y Económicas._https://www.academia.edu/9958993/ Interaprendizaje_de_Estad\%C3\%ADstica_B\%C3\%A1sica

Venezuela. (2001). Norma venezolana COVENIN 1756:2001. Edificaciones Sismorresistentes Parte 1. http://www.sencamer.gob.ve/sencamer/normas/1756-1-o1.pdf

Wilches Chaux, G. (1993). La vulnerabilidad global. En: Maskrey, A (Ed.), Los desastres no son naturales. La Red de estudios sociales en prevención de desastres en América Latina (LA RED). 